\title{
Positron Annihilation Spectroscopy of Vacancy-Type Defects Hierarchy in Submicrocrystalline Nickel during Annealing
}

\author{
Pavel V. Kuznetsov ${ }^{1,2, a)}$, Yuri P. Mironov ${ }^{1, \text { b) }}$, Aleksey I. Tolmachev ${ }^{1, c)}$, \\ Tanzilya V. Rakhmatulina, d), Yuri S. Bordulev ${ }^{2, \text { e) }}$, Roman S. Laptev ${ }^{2, \text { f) }}$, \\ Andrey M. Lider ${ }^{2, g}$, Andrey A. Mikhailov², and Alexander V. Korznikov, h) \\ ${ }^{1}$ Institute of Strength Physics and Materials Science SB RAS, Tomsk, 634055, Russia \\ ${ }^{2}$ National Research Tomsk Polytechnic University, Tomsk, 634050, Russia \\ ${ }^{3}$ Institute for Metals Superplasticity Problems RAS, Ufa, 450001, Russia \\ ${ }^{a)}$ Corresponding author: kpv@ispms.tsc.ru \\ b) myp@ispms.tsc.ru \\ c) tolmach@ispms.tsc.ru \\ d)rakhmatulina.tanya@gmail.com \\ e)bordulev@gmail.com \\ f) laptev.roman@gmail.com \\ g)lider@tpu.ru \\ h) korznikov@imsp.ru
}

\begin{abstract}
Positron annihilation and X-ray diffraction analysis have been used to study submicrocrystalline nickel samples prepared by equal channel angular pressing. In the as-prepared samples the positrons are trapped at dislocationtype defects and in vacancy clusters that can include up to 5 vacancies. The study has revealed that the main positron trap centers at the annealing temperature of $\Delta T=20^{\circ} \mathrm{C}-180^{\circ} \mathrm{C}$ are low-angle boundaries enriched by impurities. At $\Delta T=$ $180^{\circ} \mathrm{C}-360^{\circ} \mathrm{C}$, the trap centers are low-angle boundaries providing the grain growth due to recrystallization in-situ.
\end{abstract}

Keywords: submicrocrystalline structure, subgrain, grain boundary, low-angle boundary, positron annihilation, dislocations, vacancy cluster, annealing

\section{INTRODUCTION}

It is well-known that in submicrocrystalline materials produced by severe plastic deformation a part of energy is accumulated as a hierarchy of defects of different dimensions, i.e boundaries of various types, dislocations, vacancies $[1,2]$. During heating of submicrocrystalline materials, a relaxation of excess energy occurs due to transformation of boundaries, reorganization of dislocations, and annealing of defects in bulk crystallites. The study of such processes is of great interest for understanding the role of various defects in the formation of the submicrocrystalline materials properties during annealing. In the present work, annealing of vacancy-type defects in submicrocrystalline nickel is studied with the use of positron annihilation and X-ray diffraction analysis.

\section{MATERIALS AND METHODS}

Submicrocrystalline nickel with the purity of 99.998 was prepared by equal channel angular pressing implementing the route $B_{\mathrm{c}}(N=4$ passes $)$ at room temperature with the consequent rolling. The samples were EDcut across the rolling direction, and then mechanically and electrolytically polished.

International Conference on Physical Mesomechanics of Multilevel Systems 2014

AIP Conf. Proc. 1623, 327-330 (2014); doi: 10.1063/1.4901488

(C) 2014 AIP Publishing LLC 978-0-7354-1260-6/\$30.00 

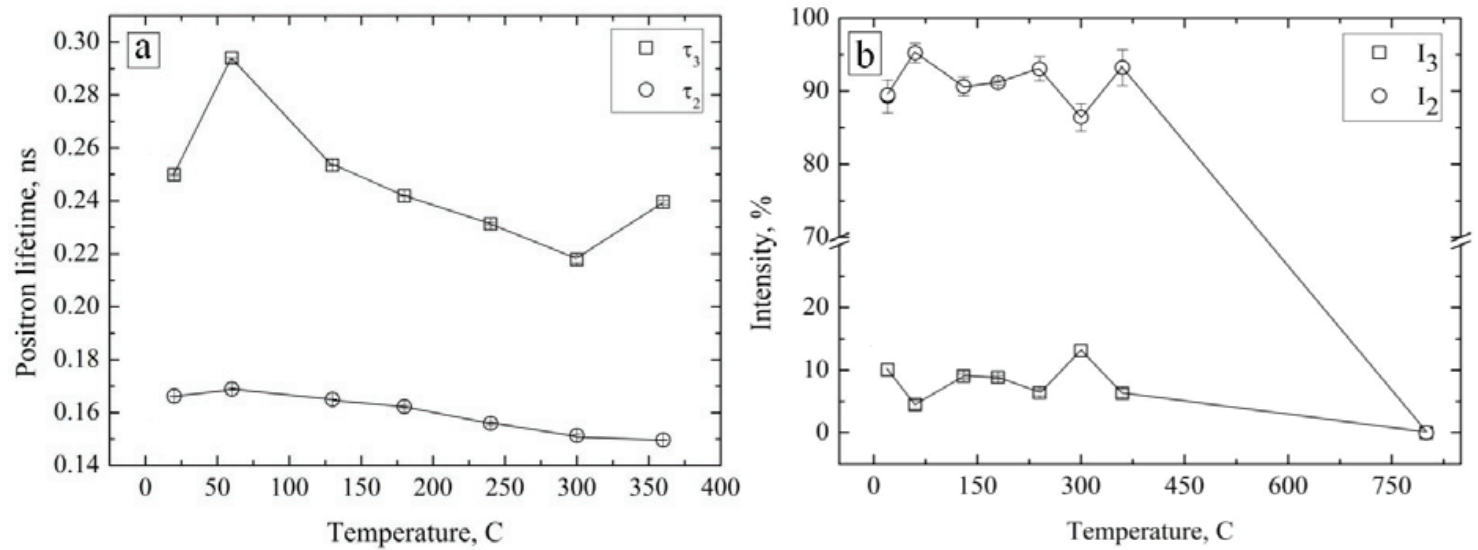

FIGURE 1. Lifetime of the components resolved in positron lifetime spectra $\tau_{2}, \tau_{3}$ (a) and the corresponding intensities $I_{2}$ and $I_{3}(\mathrm{~b})$ as the function of the annealing temperature

Isochronal annealing of the samples was carried out at temperatures varying from room temperature to $360^{\circ} \mathrm{C}$ with the step of $60^{\circ} \mathrm{C}$ and the annealing time of 15 minutes at every temperature value. Several samples were subjected to recrystallization at $800^{\circ} \mathrm{C}$ for 1 hour. X-ray diffraction analysis of the samples was performed on the DRON-7 X-ray diffractometer ("NANOTECH" of ISPMS SB RAS) with a Co-K $\mathrm{K}_{\alpha}$ source using symmetric reflection scheme without a monochromator.

Positron annihilation was studied by measuring positron lifetime spectra and Doppler broadening of the annihilation line. Positron lifetime spectra were measured using a spectrometer with the time resolution of $\sim 240$ ps. Doppler broadening spectra of the annihilation line were measured by a spectrometer based on the semiconductor detectors with the resolution of 0.8 and $1.8 \mathrm{keV}$ for the peaks of 122 and $1332 \mathrm{keV}$, respectively. The detection efficiency was $\sim 20 \%{ }^{44} \mathrm{Ti}$ isotope with an activity of $24.5 \mu \mathrm{Ci}$ was used as the source of positrons. For every sample, we obtained three positron lifetime spectra having $5 \cdot 10^{6}$ events in a spectrum. Positron lifetime spectra and Doppler broadening spectra of the annihilation line were processed by a special software.

\section{RESULTS AND DISCUSSION}

X-ray diffraction analysis of the samples reveals that the plains peak intensity (111) in as-prepared submicrocrystalline nickel is very small, that testifies the formation of the pronounced deformation texture. Annealing of the samples at $\Delta T=20^{\circ} \mathrm{C}-360^{\circ} \mathrm{C}$ does not lead to a significant change of the texture. Annealing of the samples at $T \sim 800^{\circ} \mathrm{C}$ causes the formation of the annealing texture with a high peak intensity of the plains (200).

Analysis of positron lifetime spectra of as-prepared submicrocrystalline nickel, annealed at $\Delta T=60^{\circ} \mathrm{C}-360^{\circ} \mathrm{C}$, enables the detection of components with specific lifetimes $\tau_{2} \sim 150-165 \mathrm{ps}$ and $\tau_{3} \sim 230-290 \mathrm{ps}$. The dependence of $\tau_{2}(\bullet)$ and $\tau_{3}(\bullet)$ and the corresponding intensities $I_{2}(\circ)$ and $I_{3}(\square)$ on the annealing temperature is depicted in Fig. 1.

Figure 1(a) shows that the component $\tau_{2}$ decreases from 165 to $150 \mathrm{ps}$ with the annealing temperature growth at $\Delta T=20^{\circ} \mathrm{C}-240^{\circ} \mathrm{C}$. The value $\tau_{2} \sim 150 \mathrm{ps}$ corresponds to the lifetime of the positrons trapped at dislocations in nickel [3]. The intensity $I_{2}$ of this component varies from $87 \%$ to $95 \%$ at $\Delta T=60^{\circ} \mathrm{C}-360^{\circ} \mathrm{C}$ (Fig. 1 (b)).

The component of positron lifetime spectra $\tau_{3} \sim 230-290$ ps demonstrates the trap of positrons at defects that have a larger free volume in comparison to single vacancies and dislocations [3-5]. Following [3-5], the value of the component $\tau_{3} \sim 230-290 \mathrm{ps}$ is considered to be typical for vacancy clusters. Using the theoretical dependence of the positron lifetime on a vacancy clusters size [6] in nickel, we have found that their sizes in the studied samples of submicrocrystalline nickel vary from $N=4$ to $N=9$ vacancies at $\Delta T=60^{\circ} \mathrm{C}-360^{\circ} \mathrm{C}$. The intensity of the corresponding component $\mathrm{I}_{3}$ varies from $5 \%$ to $14 \%$. The similar effect of the vacancy clusters size alteration during annealing of submicrocrystalline nickel, produced by high pressure torsion, has been observed in [3].

Doppler broadening spectra of the annihilation line allow to study the momentum distribution of electrons [7]. Analysis of Doppler broadening spectra of the annihilation line involves $S$ and $W$, which are the parameters of a line shape corresponding to annihilation of positrons with valence and core electrons, respectively. 


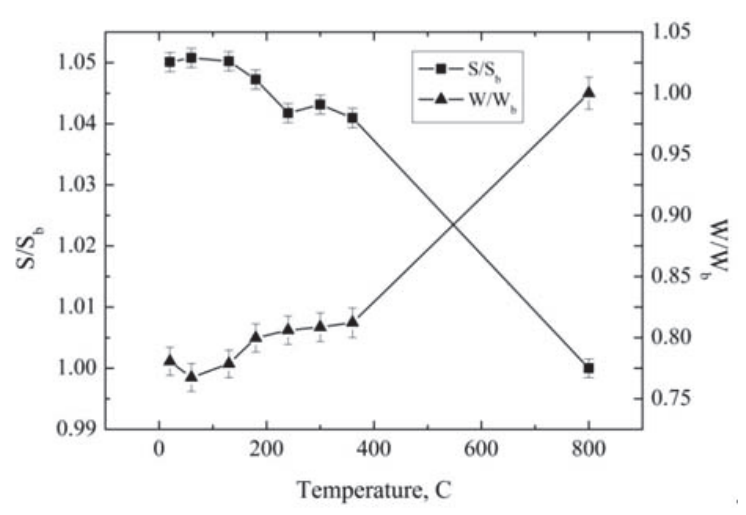

FIGURE 2. Dependence of $S$ and $W$ on the submicrocrystalline nickel annealing temperature. Parameters are respectively normalized to $S_{\mathrm{b}}, W_{\mathrm{b}}$ for the recrystallized samples

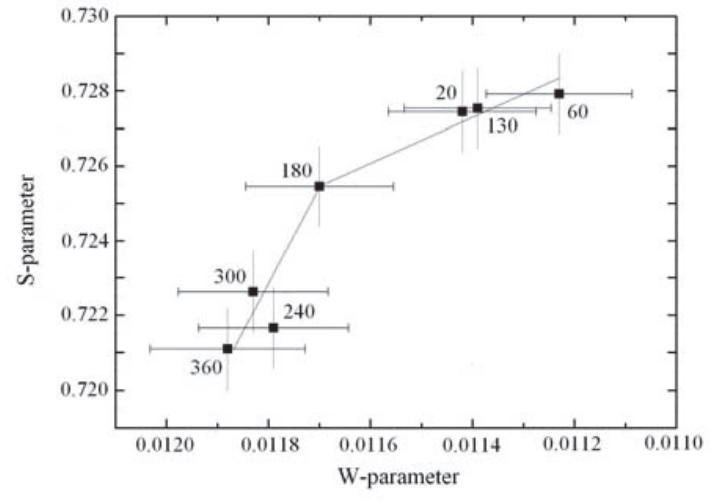

FIGURE 3. Dependence of $S$-parameter on $W$-parameter for the submicrocrystalline nickel samples annealed at various temperatures

Parameters $S$ and $W$ are defined as the ratio of a number of annihilation events in center/wings of the distribution plot to the area of the annihilation peak of $511 \mathrm{keV}$, respectively [7]. When positrons are trapped at vacancy defects the probability of their annihilation with valence electrons possessing low momentum values increases, while the probability of annihilation with core electrons with high momentum values decreases. This leads to growing $S$ and decreasing $W$, which is also affected by chemical environment at the site of positron annihilation.

Parameters $S$ and $W$ depend on both concentration and type of a defect [7]. Authors of [8] suggest a graphical method to determine a defect parameter $\mathrm{R}$, which is a parameter that does not depend on the defect concentration, but is determined only by its type. According to [8], inclination of a straight line of the function $S=f(W)$ plotted using experimentally measured values of $S_{\mathrm{d}}, W_{\mathrm{d}}$ for the series of samples yields $R$, the parameter of a defect being a dominant positron trap. Thus, the change of the inclination of a straight line $S_{\mathrm{d}}, W_{\mathrm{d}}$ of a dependence $S=f(W)$ means the change of the parameter $R$ and the dominant positron trapping defect.

Figure 2 shows the normalized dependencies $S / S_{\mathrm{b}}$ and $W / W_{\mathrm{b}}$ on the submicrocrystalline nickel annealing temperature, where $S_{\mathrm{b}}, W_{\mathrm{b}}$ are the parameters values for the recrystallized samples without defects.

Figure 3 shows the plot $S=f(W)$ for the studied samples.

High values of $S$-parameter and low values of $W$-parameter for the as-prepared samples of submicrocrystalline nickel (Fig. 2) testify the positron trapping at vacancy defects. Decrease of $S$-parameter at $\Delta T=120^{\circ} \mathrm{C}-240^{\circ} \mathrm{C}$ indicates the annealing of vacancy defects. Comparing to $S$-parameter, $W$-parameter starts changing at a lower annealing temperature of $\Delta T=20^{\circ} \mathrm{C}-180^{\circ} \mathrm{C}$ (Fig. 2). At $\Delta T=180^{\circ} \mathrm{C}-360^{\circ} \mathrm{C}$, the growth rate of $W$-parameter decreases reaching the maximum value for the recrystallized samples (Fig. 2).

Figure 3 shows that data points of $S=f(W)$ plot can generate two straight line segments having different inclination to the axes. This enables the determination of two values of R-parameter, that testifies the difference of defect types which prevail as the positron traps in submicrocrystalline nickel at the annealing temperatures of $\Delta T=20^{\circ} \mathrm{C}-180^{\circ} \mathrm{C}\left(R_{1}\right)$ and $\Delta T=180^{\circ} \mathrm{C}-360^{\circ} \mathrm{C}\left(R_{2}\right)$.

Authors of [9] have used scanning tunnel microscopy for the analysis of grain-subgrain structure of the samples studied in the present work and have revealed different processes at the annealing temperatures of $\Delta T=20^{\circ} \mathrm{C}-180^{\circ} \mathrm{C}$ and $\Delta T=180^{\circ} \mathrm{C}-360^{\circ} \mathrm{C}$. At $\Delta T=20^{\circ} \mathrm{C}-180^{\circ} \mathrm{C}$, the authors of [9] have found nonequiaxiality decrease and grains refinement. At a smaller scale level, the growth of subgrains sizes with the maximum of $\sim 60 \mathrm{~nm}$ has been observed after annealing. This agrees with the growth of coherent scattering regions measured using X-ray diffraction analysis.

At $\Delta T=240^{\circ} \mathrm{C}-360^{\circ} \mathrm{C}$ there is a growth of submicrocrystalline nickel grains [9] along with a sharp decrease of microstrain identified with the use of X-ray diffraction analysis, that indicates the initiation of recrystallization. According to [7], positrons are trapped at vacancy defects of a metal if an average distance between them is smaller than a mean free path of thermalized positrons. According to [3], the mean positron diffusion length for nickel at room temperature is $l_{\text {term }} \sim 150 \mathrm{~nm}$. Thus, it is evident that annihilation characteristics of positrons at $\Delta T=20^{\circ} \mathrm{C}-$ $180^{\circ} \mathrm{C}$ and $\Delta T=180^{\circ} \mathrm{C}-360^{\circ} \mathrm{C}$ are related to the processes in the submicrocrystalline structure with participation of positron-sensitive defects. At $\Delta T=20^{\circ} \mathrm{C}-180^{\circ} \mathrm{C}$ such defects are low-angle boundaries having the positron characteristic lifetime similar to that in dislocations [4]. Low-angle boundaries cause the growth of submicrocrystalline nickel subgrains within the limits of $\Delta l \sim 35-60 \mathrm{~nm}$ [9] with the annealing temperature increase. 
This explains the presence of component $\tau_{2} \sim 160 \mathrm{ps}$ in positron lifetime spectra of submicrocrystalline nickel (Fig. 1) and the growth of $W$-parameter demonstrating the change of chemical environment in the site of positron annihilation (Fig. 2). Apparently, the chemical composition of low-angle boundaries alters due to the entrainment of impurities from bulk crystallite during the subgrain growth.

Since the deformation texture remains at the annealing temperatures of $\Delta T=20^{\circ} \mathrm{C}-360^{\circ} \mathrm{C}$, the recrystallization of submicrocrystalline nickel at $T \geq 240^{\circ} \mathrm{C}$ is not associated with the appearance of grains with new orientation. In positron lifetime spectra (Fig. 1) there is no lifetime component $\tau_{\mathrm{g} . \mathrm{b}} \sim 290 \mathrm{ps}$ of the positrons trapped at high-angle boundaries in $\mathrm{Ni}$ [4]. Such results can be explained on the assumption that so-called recrystallization in-situ occurs at $T \geq 240^{\circ} \mathrm{C}$ in submicrocrystalline nickel. The recrystallization is related to the displacement of low-angle boundaries. It has been recently detected by the authors of [10] for the submicrocrystalline nickel samples prepared by equal channel angular pressing. This allows concluding that the dominant centers of positron trapping in submicrocrystalline nickel at $T \geq 240^{\circ} \mathrm{C}$ are low-angle boundaries.

Thus, the study results allow the following conclusions.

1. In submicrocrystalline nickel prepared by equal channel angular pressing method implementing the route $\mathrm{B}_{\mathrm{c} 4}$ positrons are trapped at dislocation defects and in vacancy clusters that include $\sim 5$ vacancies.

2. The dominant centers of positron trapping at $\Delta T=20^{\circ} \mathrm{C}-180^{\circ} \mathrm{C}$ are low-angle boundaries enriched by impurities. Displacement of low-angle boundaries and the subgrain growth are accompanied by alteration of their chemical composition due to the entrainment of impurities from bulk crystallites causing the change of the $\mathrm{W}$ and $\mathrm{R}$ parameters.

3. The dominant centers of positron trapping at the annealing temperatures of $\Delta T=180^{\circ} \mathrm{C}-360^{\circ} \mathrm{C}$ are low-angle boundaries providing recrystallization in-situ and grain growth in submicrocrystalline nickel.

4. Vacancy clusters show a high stability in the submicrocrystalline nickel structure. With annealing temperature increase within the limits of $\Delta T=20^{\circ} \mathrm{C}-300^{\circ} \mathrm{C}$, the size of clusters varies from 4 to 9 vacancies.

\section{REFERENCES}

1. R. Z. Valiev and I. V. Alexandrov, Nanostructured Materials Produced by Severe Plastic Deformation (Logos, Moscow, 2000).

2. S. S. Gorelik, S. V. Dobatkin, and L. M. Kaputkina, Recrystallization of Metals and Alloys (MISIS, Moscow, 2005).

3. J. Cizek, I. Prochazka, M. Cieslar, I. Stulikova, F. Chmelik, and R. Islamgaliev, Phys. Stat. Sol. A 191, 391 (2002).

4. T. E. M. Staab, R. Krause-Rehberg, and B. Kieback, J. Mater. Sci. 34, 3833 (1999).

5. G. Dlubekt, O. Brummert, N. Meyendorfi, P. Hautojarvi, A. Vehanent, and J. Yli-Kauppila, J. Phys. F: Metal Phys. 9, 1961 (1979).

6. M. J. Puska and R. M. Nieminen, J. Phys. F: Metal Phys., 333 (1983).

7. R. Krause-Rehberg and H. S. Leipner, Positron Annihilation in Semiconductors, Defect Studies (Springer, Berlin, 1999).

8. L. Liszkay, C. Corbel, L. Baroux, P. Hautojarvi, M. Bayhan, A. W. Brinkman, and S. Tatarenko, Appl. Phys. Lett. 64, 1380 (1994).

9. P. V. Kuznetsov, I. V. Petrakova, T. V. Rakhmatulina, A. A. Baturin, and A. V. Korznikov, Ind. Lab. 78, 26 (2012).

10. S. V. Divinski, G. Reglitz, M. Wegner, M. Peterlechner, and G. Wilde, J. Appl. Phys. 115, 113503-1 (2014). 